\title{
Duration of Untreated Psychosis: An Important Prognostic Indicator
}

\author{
Ajay Pal Singh* \\ Department of Psychiatry, California Northstate University College of Medicine, USA
}

*Corresponding author: Ajay Pal Singh, Department of Psychiatry, California Northstate University College of Medicine, USA, Fax: 844-427-2226; Email: ajpsingh@ucdavis.edu

Received date: December 12, 2017; Accepted date: December 12, 2017; Published date: December 18, 2017

Citation: Singh AP (2018) Duration of Untreated psychosis: An Important Prognostic Indicator. Dual Diagn Open Acc Vol.3 No.1: 2.

Copyright: (02018 Singh AP. This is an open-access article distributed under the terms of the Creative Commons Attribution License, which permits unrestricted use, distribution, and reproduction in any medium, provided the original author and source are credited.

\section{Editorial}

'Schizophrenia arguably the worst disease affecting mankind' [1]. Psychosis is a thought disorder which may result in varying degrees of loss of contact with reality manifesting primarily as hallucinations, delusions and paranoia. The usual age of onset is late adolescence or early 20s. Early onset psychosis is accompanied with diagnostic challenges and complexity.

It is usually difficult to predict and ascertain a cause and progression of the first episode of psychosis (FEP). It is understood to be a combination of genetic, biological and environmental factors. A thorough medical and psychological evaluation is a key to the diagnosis and management of psychosis. With the advancement of treatment and the availability of newer antipsychotics the rates of remission of psychosis have improved significantly. In one study, patients were followed up for six years and $15.6 \%$ had complete remission after the first episode [2].

Approximately 100,000 people experience first episode of psychosis every year [3]. The Neurodegenerative process progresses intensely and rapidly in the first years of the onset of psychosis. Several studies have postulated that the delays in initiating treatment for psychosis negatively affect the outcome and prognosis including poorer response to treatment, failure to attain remission and long-term decline in functioning [4-6].

In the US, the average delay between psychotic symptom onset and starting treatment is 17 months [7]. Duration of psychosis (DUP) of 3 months or less is optimal for treatment response [8]. National Institute of Mental Health (NIMH) is taking initiatives to support identification and referral of FEP cases. Reducing DUP from current levels of greater than one year to the recommended standard of less than three months [8].

Patients with Schizophrenia have a lower life expectancy than general population and are at a higher risk of developing heart disease and other metabolic diseases. This is a result of the side effects of antipsychotic medications and poor lifestyle behaviors. These factors are modifiable and can have better outcomes with early and aggressive treatment of psychosis.

In addition cultural, social, religious and economic factors, the barriers to early intervention include lack of understanding and attitudes in patients and their families, the associated stigma, access to specialized healthcare, the symptoms of the disease itself act as barriers in accessing treatment [9].

Early detection rates can increase by improving clinician training, easing referral process and the, early availability of treatment appointments, use of telemedicine for underserved areas, using multimodal treatment modalities psychotherapy and psycho-pharmcology. The early results from new treatment approaches like virtual treatment and Avatar therapy are promising [10]. In Avatar therapy, patient with the help of computer programs designs a face that matches one of the auditory hallucination. The therapist communicates with the patient through this Avatar and assists the patient to relax to give a facial recognition to their voice.

The first episode of psychosis requires the same amount of aggression in treatment as other potential life threatening disorders. As Dr. H. Nasrallah in his editorial suggested 'For firstepisode psychosis, psychiatrists should behave like cardiologists' [11]. He has drawn analogies between myocardial infraction and FEP suggesting administering long-acting injectable at FEP to avoid recurrence of psychosis. Reducing the duration of untreated psychosis to by identification and removing of barriers and promoting early management of first episode psychosis will have a direct positive outcome on long-term recovery, quality of life and longevity. Early identification and intervention is the key to prevent the second episode.

\section{References}

1. Where next with psychiatric illness? (1988) Nature 336: 95-96 Available at https://www.nature.com/articles/336095a0

2. Rosen K, Garety P (2005) Predicting Recovery From Schizophrenia: A Retrospective Comparison of Characteristics at Onset of People With Single and Multiple Episodes. Schizophr Bull 31: 735-750.

3. McGrath J, Saha S, Chant D, Welham J (2008) Schizophrenia: a concise overview of incidence, prevalence, and mortality. Epidemiol Rev 30: 67-76.

4. Harris MG, Henry LP, Harrigan SM, Purcell R, Schwartz OS, et al. (2005) The relationship between duration of untreated psychosis and outcome: An eight-year prospective study. Schizophr Res 79: 85-93. 
5. Falloon IR, Kydd RR, Coverdale JH, Laidlaw TM (1996) Early detection and intervention for initial episodes of schizophrenia. Schizophr Bull 22: 271-282.

6. De Quardo JR (1998) Pharmacologic treatment of first-episode schizophrenia: early intervention is the key to the outcome. Journ Clin Psych 59: 9-17.

7. Rosenheck RA, Azrin ST, Goldstein AB, Severe J, Kane JM, et al. (2015) Duration of Untreated Psychosis in Community Treatment Settings in the United States. Psychiatr Serv 66: 753-756.

8. Bertolote J, Mcgorry P (2005) Early intervention and recovery for young people with early psychosis: consensus statement. $\mathrm{Br} \mathrm{J}$ Psychiatry 187: s116-s119.
9. Consensus Statement on Principles and Practice in Early Psychosis (2002) In Implementing Early Intervention in Psychosis: A Guide to Establishing Early Intervention in Psychosis: (eds J.Edwards \& P.D. McGorry), London pp.145-155.

10. Craig TKJ, Rus-Calafell Ma, PhD, Ward T, Julian P Leff, et al. (2017) AVATAR therapy for auditory verbal hallucinations in people with psychosis: a single-blind, randomised controlled trial. The Lancet Psychiatry.

11. Henry Nasrallah (2017) For first-episode psychosis, psychiatrists should behave like cardiologists. Curr Psychiatry 8: 4-7. 